\title{
Association between estrogen receptor $\alpha$ polymorphisms and equol production, and its relation to bone mass
}

\author{
HIRONOBU KATSUYAMA ${ }^{1}$, MASAYUKI ARII ${ }^{2}$, MASAFUMI TOMITA ${ }^{3}$, KAZUO HIDAKA ${ }^{4}$, \\ YOKO WATANABE ${ }^{4}$, YOSHIE TAMECHIKA ${ }^{5}$, TOSHIKO OKUYAMA ${ }^{3}$, SHIGEKO FUSHIMI $^{1}$, \\ NORIKO MAEDA ${ }^{6}$, TSUTOMU HIGASHIMURA ${ }^{6}$, MASAO FUKUNAGA $^{7}$ and KIYOFUMI SAIJOH ${ }^{8}$ \\ Departments of ${ }^{1}$ Public Health, ${ }^{3}$ Medical Toxicology, ${ }^{4}$ Biochemistry, ${ }^{5}$ Clinical Laboratory, ${ }^{7}$ Nuclear Medicine, \\ Kawasaki Medical School, Kurashiki 701-0192; ${ }^{2}$ Research and Development Division, Kikkoman Corporation, \\ Tokyo 105-8428; ${ }^{6}$ Kosei Hospital, Kakogawa 675-0005; ${ }^{8}$ Department of Hygiene, Kanazawa University \\ School of Medicine and Graduate School of Medical Sciences, Kanazawa 920-8640, Japan
}

Received December 11, 2008; Accepted February 23, 2009

DOI: 10.3892/ijmm_00000194

\begin{abstract}
To investigate the relationship between estrogen receptor polymorphisms and equol production and its effect on bone turnover, 139 workers (mean age $38.3 \pm 11.1$ years) in Japan were recruited. Bone mineral density (BMD), bone turnover markers, and serum equol were measured at a health examination. DNA samples were prepared to detect the estrogen receptor $\alpha(\mathrm{ER} \alpha)$ polymorphism and were digested by $P v u I I$. The number of equol producers was 57 . No statistically significant differences were observed in bone mineral density and bone turnover markers between each ER $\alpha$ polymorphism and equol production. Since the adjusted odds ratio indicated that interaction itself decreased the risk of osteosonoassessment index (OSI) reduction using logistic regression analysis, further analysis was performed divided by each ER $\alpha$ polymorphism. Although the crude odds ratio showed no relationship between equol producers and non-producers, the adjusted odds ratio showed that equol producers with ER $\alpha$ pp had a significantly decreased risk of OSI reduction. Although this study was cross-sectional, both equol production and $\mathrm{ER} \alpha$ polymorphism are closely associated with each other in relation to BMD.
\end{abstract}

\section{Introduction}

Bone mineral density (BMD), one of the most significant predictors of osteoporotic fracture, is influenced by both environmental and genetic factors (1). Although osteoporosis

Correspondence to: Dr Hironobu Katsuyama, Department of Public Health, Kawasaki Medical School, 577 Matsushima, Kurashiki 701-0192, Japan

E-mail:katsu@med.kawasaki-m.ac.jp

Key words: equol producer, estrogen receptor $\alpha$ polymorphism, osteosono-assessment index, bone turnover markers, logistic regression analysis results in inadequate skeletal strength predisposing its sufferers to fracture, most osteoporotic patients are diagnosed after the occurrence of fractures because osteoporosis itself lacks specific symptoms. Even if osteoporosis is diagnosed before a fracture occurs, it is difficult to recover BMD in a short period of time. In females, BMD reaches its maximum in adolescence and decreases gradually until menopause, after which it decreases dramatically (2). Since reduction in BMD due to menopause is unavoidable, it seems important to achieve the maximum possible level of BMD in adolescence and to prevent BMD reduction before menopause.

Estrogen deficiency plays a major role in the pathogenesis of postmenopausal osteoporosis. Estrogen deficiency is characterized by increased bone resorption and a remodeling imbalance (3). The restriction fragment length polymorphisms on intron I of the estrogen receptor $\alpha(\mathrm{ER} \alpha)$ gene are also supposed to affect BMD since the Px haplotype, in which $\mathrm{P}$ lacks a $P v u \mathrm{II}$ site and $\mathrm{x}$ possesses an $\mathrm{XbaI}$ site, shows a significant association with low BMD (4). The BMD of the femur and lumbar spine of healthy young men displayed the same tendency (5). On the other hand, recent meta-analysis data suggest that $\mathrm{XX}$ of ER $\alpha$ polymorphism may be related to high BMD and low fracture risk $(6,7)$. Thus, the effect of $\mathrm{ER} \alpha$ polymorphism on BMD is still controversial. However, it appears that women with the $\mathrm{P}$ allele may benefit more from the protective effect of postmenopausal hormone replacement therapy (HRT) than those with the pp homozygote (8). In addition, this $P v u I I$ site is located in a potential binding site for $\mathrm{B}$ myb transcription factor (9). Thus, this $\mathrm{P} / \mathrm{p}$ polymorphism was employed in the present study.

Soy is part of the traditional diet of Asians, and epidemiological data indicate that Asian people have a lower rate of osteoporotic fractures, cardiovascular diseases, postmenopausal symptoms, and certain types of cancers than Western populations (10). Soy isoflavones are often referred to as weak estrogens because genistein is a relative potent agonist for ERß (11). Soy isoflavones, such as genistein, daidzein, and glycitein show estrogenic activity so they are also called phytoestrogens. In humans, equol is produced from daidzein in the gastrointestinal tract (12). Equol is structurally similar 
to 173 -estradiol, which has an affinity for estrogen receptors so that it possesses estrogenic activity. Dietary equol exerts mild estrogenic effects in the pituitary, uterus, mammary glands, liver and fat tissue, but shows limited bone sparing effects in ovariectomized rats (12). Moreover, the relationship between ER receptor polymorphisms and equol production and its effect on bone turnover is still uncertain.

In the present study, we investigated the relationship between ER polymorphisms and equol production and the effects on bone turnover.

\section{Subjects and methods}

Subjects. A total of 139 workers at local hospitals in Japan were recruited with informed consent. The subjects had a mean age of $38.3 \pm 11.1$ years and 22 were male. Both premenopausal and perimenopausal women were included in the study.

This study was approved by the Medical Ethics Committee of Kawasaki Medical School and Kawasaki Medical School Hospital (no. 100).

Lifestyle factors. The usual dietary intake was assessed using a food frequency questionnaire according to an 11-foodgroup classification (13). This questionnaire was designed and validated previously to measure frequent food intake for seven days. Daily dietary intakes were calculated from this seven-day weighed-food intake questionnaire. We used the soybean intake calculated by this questionnaire.

The subjects had their heights and weights measured wearing light clothing, and their body mass indexes (BMI) were calculated.

Bone mineral density measurement. Bone mineral density was measured by an ultrasound method to obviate X-ray exposure. The speed of sound, transmission index, and osteosonoassessment index (OSI) at the calcaneus were measured by using quantitative ultrasound (QUS) AOS-100 (Aloka Co., Ltd., Tokyo). We used OSI as the bone mineral parameter.

Biochemical markers of bone turnover. The second morning urine specimens were collected, and blood samples were obtained at the same time as QUS measurement. Both serum and urine specimens were stored at $-20^{\circ} \mathrm{C}$ until measurement. Serum bone-specific alkaline phosphatase (BAP) and serum $\gamma$-carboxylated (Gla) osteocalcin were measured as bone formation markers. Gla was measured using the Gla-type osteocalcin EIA kit (Takara, Osaka, Japan). Osteocalcin correlates well with bone formation, but is readily subject to rapid degradation in serum (14). Since not only Gla-osteocalcin and undercarboxylated osteocalcin (uc-OC) but also fragments of various sizes of them coexist in the circulation, different immunoassays have yielded varying results (15). In the present study, a kit utilizing an antibody specific to $17 \mathrm{Gla}$ and its adjacent region was used to isolate Gla. Although $\gamma$ carboxylation preferentially occurred at residues 21 and 24 (16), 17Gla is essential for a Ca-dependent conformational transition (17), indicating that the $17 \mathrm{Gla}$-specific antibody recognizes fully $\gamma$-carboxylated osteocalcin to be active. Deoxypyridinoline (DPD) and type I collagen cross-linked $\mathrm{N}$-telopeptides (NTX) in urine were measured as bone

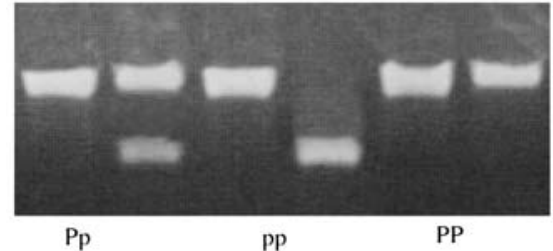

Figure 1. Representative types of polymorphisms in the estrogen receptor $\alpha$ $(\mathrm{ER} \alpha)$ gene.

resorption markers. Both were corrected by creatinine in urine. The menstrual cycle of each subject was not considered as the effects of the menstrual cycle on bone turnover are thought to be small (18).

Measurement of equol. Serum equol was measured by high performance liquid chromatography (HPLC) according to the method of Hidaka et al (19). Although many studies suggest that Bifidobacterium, Escherichia coli, Bacteroides ovatus, Ruminococcus productus and Streptococcus intermedius could be involved in daidzein metabolism $(20,21)$, the main bacteria responsible for daidzein have not been definitely identified (22). In humans, only $30-50 \%$ of individuals are capable of producing equol from daidzein (23). An equol producer was defined as one who had a serum concentration of equol $>4 \mathrm{ng} / \mathrm{ml}$.

ERa polymorphism. Blood samples were obtained at health examination, and genomic DNA was extracted from leukocytes. Intron 1 of the ER gene, where the $P v u I I$ digestion site is located, was amplified using polymerase chain reaction (PCR) (24). After amplification using a set of primers (forward: CTGCCACCCTATCTGTATCTTTTCCTATTCTCC and reverse: TCTTTCTCTGCCACCCTGGCGTCGATTATC TGA), the products were digested with $P v u$ II: $\mathrm{p}$ but not $\mathrm{P}$ was digested. Fig. 1 shows the ER $\alpha$ polymorphism types.

Statistical analysis. One-way analysis of variance (ANOVA) with Fisher's PLSD as a post hoc test was performed to compare differences in lifestyle factors, biochemical markers of bone turnover, and OSI for each ER $\alpha$ polymorphism and equol producer. Two-way ANOVA and a logistic regression test were performed to examine the interaction between ER $\alpha$ polymorphism and equol production statuses. When significant differences were detected, contrast analyses were performed using Sheffe's multiple comparison test. Odds ratios as estimated risk with $95 \%$ confidence intervals were calculated by logistic regression using JMP 7.0 (SAS Corp.).

\section{Results}

Lifestyle factors, equol production, and ER polymorphisms of subjects. No statistical difference in mean age was observed between males and females (Table I). The daily intake of soybean and soy-related foods was $\sim 60 \mathrm{~g}$, which is the same as the Japanese average (25). The mean BMI was $\sim 22$, indicating that most subjects were not obese. Bone biochemical markers and OSI of all subjects were within normal limits, indicating that the subjects, including the postmenopausal women, were 
Table I. Characteristics of subjects.

\begin{tabular}{|c|c|c|c|c|c|c|}
\hline & \multirow[b]{2}{*}{ Total } & \multicolumn{3}{|c|}{ Divided by ER $\alpha$ polymorphism } & \multicolumn{2}{|c|}{ Divided by equol production } \\
\hline & & $\mathrm{PP}$ & $\mathrm{Pp}$ & $\mathrm{pp}$ & Producer & Non-producer \\
\hline Number of subjects & 139 & 22 & 68 & 49 & 57 & 82 \\
\hline $\begin{array}{l}\text { Mean age } \\
\text { Male }(n=22) \\
\text { Female }(n=117)\end{array}$ & $\begin{array}{l}38.3 \pm 11.1 \\
38.0 \pm 13.4 \\
38.4 \pm 10.7\end{array}$ & $38.9 \pm 12.0$ & $39.2 \pm 11.5$ & $36.9 \pm 10.2$ & $39.9 \pm 11.2$ & $37.2 \pm 11.0$ \\
\hline Soybean intake (g/D) & $61.0 \pm 38.0$ & $65.9 \pm 47.1$ & $66.2 \pm 37.2$ & $51.2 \pm 33.0$ & $65.9 \pm 44.8$ & $57.3 \pm 31.9$ \\
\hline BMI & $21.9 \pm 3.0$ & $20.9 \pm 2.7$ & $22.5 \pm 3.1$ & $21.5 \pm 2.7$ & $22.5 \pm 3.1$ & $21.5 \pm 2.8$ \\
\hline Gla (ng/ml) & $4.87 \pm 2.44$ & $5.23 \pm 3.00$ & $4.90 \pm 2.63$ & $4.67 \pm 1.86$ & $5.06 \pm 2.27$ & $4.73 \pm 2.56$ \\
\hline uc-OC (ng/ml) & $2.00 \pm 1.02$ & $2.29 \pm 1.21$ & $1.79 \pm 0.89$ & $2.15 \pm 1.06$ & $1.82 \pm 0.97$ & $2.12 \pm 1.05$ \\
\hline $\mathrm{BAP}(\mathrm{U} / \mathrm{l})$ & $23.7 \pm 8.4$ & $26.4 \pm 10.9$ & $23.5 \pm 7.9$ & $22.6 \pm 7.7$ & $22.9 \pm 8.4$ & $24.2 \pm 8.4$ \\
\hline $\mathrm{DPD}(\mathrm{nM} / \mathrm{mM} \mathrm{Cr})$ & $6.03 \pm 2.10$ & $6.79 \pm 2.85$ & $5.95 \pm 1.83$ & $5.81 \pm 2.03$ & $6.15 \pm 1.94$ & $5.95 \pm 2.21$ \\
\hline NTx/Cr (nM BCE/mM Cr) & $13.9 \pm 4.1$ & $12.5 \pm 5.9$ & $13.7 \pm 3.7$ & $14.7 \pm 3.5$ & $13.7 \pm 4.5$ & $14.0 \pm 3.8$ \\
\hline OSI & $2.66 \pm 0.29$ & $2.65 \pm 0.27$ & $2.67 \pm 0.31$ & $2.67 \pm 0.28$ & $2.67 \pm 0.30$ & $2.66 \pm 0.28$ \\
\hline
\end{tabular}

Gla, carboxylated osteocalcin; uc-OC, undercarboxylated osteocalcin; BAP, bone-specific alkaline phosphatase; NTX, N-terminal cross linked telopeptide; DPD, deoxypiridinoline; OSI, osteosono-assessment index. No statistical difference was observed in each ERa polymorphism or equol production.

thought to be healthy and did not have osteoporosis or bone disease.

When ER $\alpha$ RFLPs were considered, in good accordance with previous reports $(4,26), \sim 35.3 \%$ of subjects had the pp homozygote, $15.8 \%$ had the PP homozygote, and $48.9 \%$ had the Pp heterozygote. The allele frequency matched the HardyWeinberg equation. Among these three groups, no statistical differences were observed in age, daily soy intake, BMI, biochemical markers of bone turnover, and OSI.

On the other hand, $57(41.7 \%)$ subjects produced equol. When subjects were divided by equol production, there were no statistical differences in age, BMI, daily soy intake, biochemical markers of bone turnover, and OSI between equol producers and non-producers. Since the baseline characteristics did not differ between these groups as well as among ER $\alpha$ polymorphism groups, the interactional effect on $\mathrm{ER} \alpha$ polymorphisms and equol production was assessed.

Univariate comparison of bone biochemical markers of bone turnover and OSI. When interactional effects on bone formation markers and OSI were assessed using two-way ANOVA (Fig. 2), no statistical differences were observed in OSI, Gla and BAP. However, uc-OC showed an interactional effect, i.e., equol non-producers with PP and equol producers with pp showed high uc-OC levels. Bone resorption markers such as DPD and NTX produced no interactional effects (Fig. 3). These results indicated that equol production and ER polymorphism were correlated with each other. The univariate analysis seemed to be insufficient to exclude confounding factors and to estimate which parameter was associated with the bone mineral density so that further multivariate analyses were performed using logistic regression analysis.
Table II. Risk determinants in the reduction of OSI.

\begin{tabular}{|c|c|c|}
\hline & $\begin{array}{l}\text { Crude odds ratio } \\
\qquad(95 \% \mathrm{CI})\end{array}$ & $\begin{array}{l}\text { Adjusted odds ratio } \\
\qquad(95 \% \mathrm{CI})\end{array}$ \\
\hline \multicolumn{3}{|l|}{$\mathrm{ER} \alpha$} \\
\hline $\mathrm{pp}$ & 1.000 (reference) & 1.000 (reference) \\
\hline $\mathrm{Pp}$ & $1.147(0.451-2.916)$ & $2.160(0.646-7.587)$ \\
\hline PP & $0.913(0.268-3.179)$ & $0.535(0.099-2.809)$ \\
\hline \multicolumn{3}{|l|}{ Equol } \\
\hline Non-producer & 1.000 (reference) & 1.000 (reference) \\
\hline Producer & $0.787(0.397-1.556)$ & $0.797(0.328-1.944)$ \\
\hline Interaction & $0.625(0.259-1.494)$ & $0.008(0.0001-0.576)^{\mathrm{a}}$ \\
\hline \multicolumn{3}{|c|}{$\begin{array}{l}\text { Odds ratios indicating that OSI was lower than } 2.66 \text { to higher than } \\
2.66 \text { were calculated according to potential risk factors. Interaction } \\
\text { was multiplied by ER } \alpha \text { and equol, and adjusted interaction itself } \\
\text { reduced the risk }\left({ }^{a} \mathrm{p}<0.05\right) \text {. Odds ratios and } 95 \% \text { confidence intervals } \\
(\mathrm{CI}) \text { were evaluated by adjusted model for age, BMI, daily soy } \\
\text { intake and bone turnover markers. }\end{array}$} \\
\hline
\end{tabular}

Logistic regression analysis and risk determinants for bone mineral loss. Since the average OSI of subjects was 2.66, a logistic regression analysis to clarify possible risk factors was performed when the OSI was $<2.66$.

The crude odds ratio showed that both ER $\alpha$ polymorphism and equol production had no association with OSI (Table II). In addition, interaction itself had also no association. The odds ratio adjusted by age, BMI, daily soy intake, and bone 

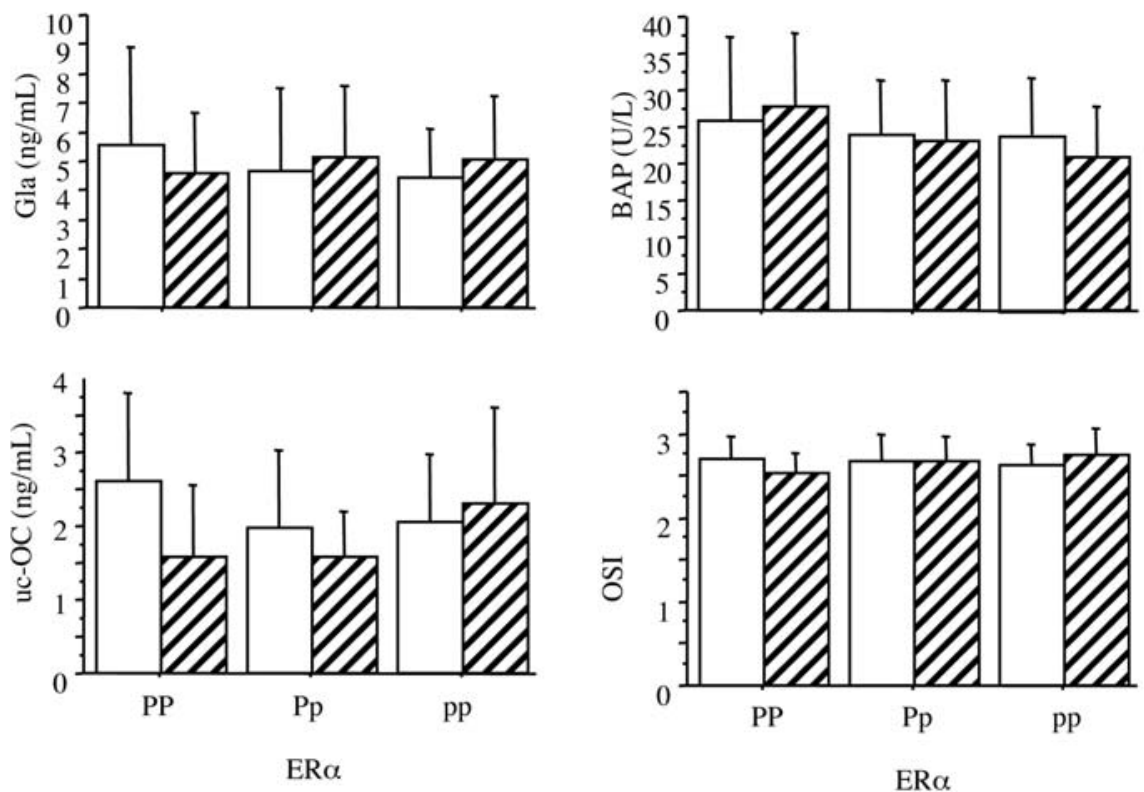

$\square$ Equol non-producer

$\boldsymbol{D}_{\text {Equol producer }}$

Figure 2. Differences in bone formation markers in each ER polymorphism and equol production. No statistical differences were observed in carboxylated osteocalcin (Gla), bone-specific alkaline phosphatase (BAP), and osteosono-assessment index (OSI). Interactional effect was shown in undercarboxylated osteocalcin (uc-OC) (two-way ANOVA, p<0.05).
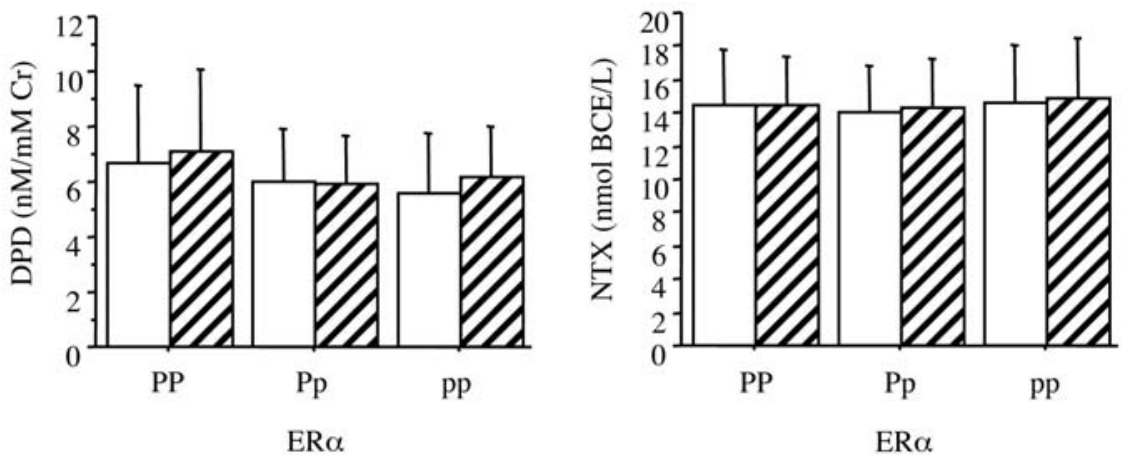

\section{$\square$ Equol non-producer \\ DEquol producer}

Figure 3. Differences in bone resorption markers in each ER polymorphism and equol production. No statistical differences were observed in deoxypiridinoline (DPD) and N-terminal cross-linked telopeptide (NTX) (two-way ANOVA).

Table III. Risk determinants in reduction of OSI divided by each ER $\alpha$ polymorphism.

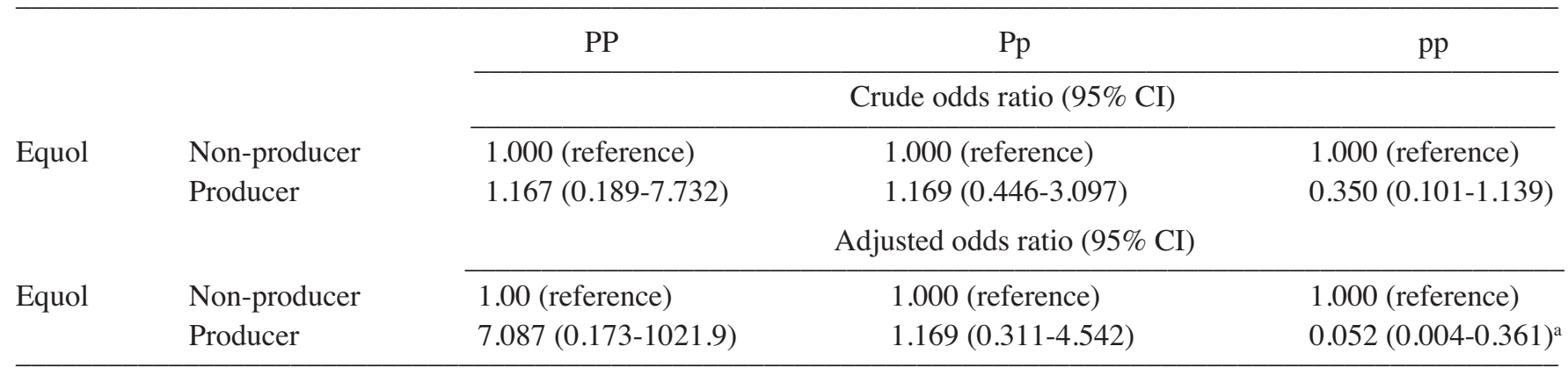

For calculation of odds ratios refer to Table II legend. 
turnover markers also showed no association between ER $\alpha$ polymorphisms and OSI, or between equol production and OSI. When the interactional effect was included as a determinant, it seemed to expose a concealed effect of a difference in ER $\alpha$ polymorphism, and the interactional effect itself reduced the risk with an odds ratio of 0.008 . Therefore, a logistic regression analysis was further performed on groups divided by ER $\alpha$ polymorphism (Table III). In the PP allele and Pp allele groups, no statistical difference in equol production was observed in both the crude odds ratio and the adjusted odds ratio. In the pp allele group, although equol production had no statistical difference in crude odds ratio, the adjusted odds ratio showed the low odds ratio of 0.052 , the $\mathrm{p}$-value of which was 0.008 .

\section{Discussion}

The risk of osteoporosis in elderly women can be assessed by peak bone mass at a young age and the rate of decline in BMD with increasing age because they lack the protective effect of estrogen after menopause. ER seems to play an important role in the maintenance of normal bone turnover, not only before menopause, but also after menopause since phytoestrogens possessing estrogenic bioactivity can be taken in the diet, especially soybeans and soy products. However, humans have ER polymorphisms displaying different affinities to bone turnover. In addition, some are equol producers who can produce highly bioavailable phytoestrogen. Thus, the exact mechanism of the bone-beneficial effect of ER polymorphisms and equol producer/non-producer status has not yet been clarified. In the present study, the effect of equol on bone turnover was analyzed according to ER polymorphisms.

The daily soy intake of the subjects was similar so that the effect of equol production could be assessed regardless of the ER $\alpha$ polymorphism. Univariate analysis showed an interactional effect only in uc-OC, indicating that equol producers with the ER $\alpha$ pp homozygote had higher uc-OC than those with the $\mathrm{P}$ allele, whose uc-OC stayed within the normal limits. Since a high level of uc-OC is associated with a high risk of bone fracture (27), multivariate analyses such as logistic regression analysis were applied to exclude the effects of confounding factors.

The crude odds ratio obtained by logistic regression analysis indicated that ER $\alpha$ polymorphisms and equol production showed no relationship to the decrease in the risk of OSI reduction. However, when odds ratios were adjusted for age, BMI, daily soy intake, and bone turnover markers, an interactional effect of $\mathrm{ER} \alpha$ polymorphisms and equol production was recognized as a preventive factor. That is, the odds ratio adjusted according to the ER $\alpha$ polymorphism showed that equol producers with the pp allele group had a decreased risk of OSI reduction. Not only did dietary equol administration to ovariectomized rats show limited bone sparing effects (12), but also long-term consumption of isoflavone-enriched foods did not affect bone metabolism in early postmenopausal women (28). However, these reports did not take ER $\alpha$ polymorphism into account. We showed a relationship between equol production and $\mathrm{ER} \alpha$ polymorphisms in bone turnover. That is, equol producers with the ER $\alpha$ pp allele might be protected from bone mineral loss compared to equol non-producers. The Px haplotype is supposed to be associated with low BMD, but HRT therapy is also reportedly more beneficial for postmenopausal women with a $\mathrm{P}$ allele than for those with the pp homozygote (8). In this study, the beneficial effect of equol production on pp homozygotes was observed in subjects including premenopausal women, postmenopausal women, and men. It is likely that the effect of equol production would be more obvious when women with the pp homozygote reach menopause.

The beneficial effects of soybean isoflavone are widely known. A randomized, placebo-controlled clinical trial showed that supplementation of $110 \mathrm{mg}$ aglycones/day is effective to significantly decrease bone resorption in postmenopausal women (29). Daily intake of isoflavones is critical because administration of $60 \mathrm{mg}$ aglycones/day relieves menopausal symptoms, administration between 50 and $110 \mathrm{mg}$ aglycones/ day reduces the risks of breast, colon, and prostate cancer, and administration of 40-60 mg aglycones/day decreases the risk of cardiovascular disease (30). On the other hand, the average intake of soy of $\sim 30 \mathrm{~g} /$ day provides nearly $40 \mathrm{mg}$ isoflavones/ day (31). In the present study, the mean soybean intake was $\sim 60 \mathrm{~g} /$ day, which was equivalent to $\sim 80 \mathrm{mg} /$ day isoflavone. This consumption might be beneficial for bone health.

In conclusion, this study indicated that equol producers with the ER $\alpha$ pp allele had a decreased risk of OSI reduction. Although this study is cross-sectional, further investigation is needed to assess the long-term effect of isoflavone supplementation on bone turnover in relation to ER polymorphism.

\section{Acknowledgements}

This study was supported, in part, by a Research Project Grant (no. 17-501) from Kawasaki Medical School.

\section{References}

1. Eisman JA: Genetics of osteoporosis. Endocr Rev 20: 788-804, 1999.

2. Yamazaki K, Kushida K, Ohkuma A, Sano M and Inoue T: Ultrasound bone densitometry of the os calcis in Japanese women. Osteoporos Int 4: 220-225, 1994.

3. Turner RT, Riggs BL and Spelsberg TC: Skeletal effects of estrogen. Endocr Rev 15: 275-300, 1994.

4. Kobayashi S, Inoue S, Hosoi T, Ouchi Y, Shiraki M and Orimo H: Association of bone mineral density with polymorphism of the estrogen receptor gene. J Bone Miner Res 11: 306-311, 1996.

5. Valimaki VV, Piippo K, Valimaki S, Loyttyniemi E, Kontula K and Valimaki MJ: The relation of the XbaI and PvuII polymorphisms of the estrogen receptor gene and the CAG repeat polymorphism of the androgen receptor gene to peak bone mass and bone turnover rate among young healthy men. Osteoporos Int 16: 1633-1640, 2005.

6. Ioannidis JP, Stavrou I, Trikalinos TA, et al: Association of polymorphisms of the estrogen receptor alpha gene with bone mineral density and fracture risk in women: a meta-analysis. J Bone Miner Res 17: 2048-2060, 2002.

7. Ioannidis JP, Ralston SH, Bennett ST, et al: Differential genetic effects of ESR1 gene polymorphisms on osteoporosis outcomes. JAMA 292: 2105-2114, 2004.

8. Herrington DM, Howard TD, Brosnihan KB, et al: Common estrogen receptor polymorphism augments effects of hormone replacement therapy on E-selectin but not C-reactive protein. Circulation 105: 1879-1882, 2002.

9. Salmen T, Heikkinen AM, Mahonen A, et al: The protective effect of hormone-replacement therapy on fracture risk is modulated by estrogen receptor alpha genotype in early postmenopausal women. J Bone Miner Res 15: 2479-2486, 2000 . 
10. Chen YM, Ho SC, Lam SSH, Ho SSS and Woo JLF: Soy isoflavones have a favorable effect on bone loss in Chinese postmenopausal women with lower bone mass: A double-blind, randomized, controlled trial. J Clin Endocrinol Metab 88: 4740-4747, 2003.

11. McCarty MF: Isoflavones made simple - genistein's agonist activity for the beta-type estrogen receptor mediates their health benefits. Med Hypotheses 66: 1093-1114, 2006.

12. Rachon D, Seidlova-Wuttke D, Vortherms T and Wuttke W: Effects of dietary equol administration on ovariectomy induced bone loss in Sprague-Dawley rats. Maturitas 58: 308-315, 2007.

13. Takahashi K, Yoshimura Y and Katashima R: Development of food frequency and intake survey by questionnaire method. Bull Shikoku Univ 5: 23-35, 1996. (In Japanese)

14. Garnero P, Grimaux M, Seguin P and Delmas PD: Characterization of immunoreactive forms of human osteocalcin generated in vivo and in vitro. J Bone Miner Res 9: 255-264, 1994.

15. Seibel MJ and Woitge HW: Basic principle and clinical applications of biochemical markers of bone metabolism. J Clin Densitom 2: 299-321, 1999.

16. Benton ME, Price PA and Suttie JW: Multi-site-specificity of the vitamin K-dependent carboxylase: In vitro carboxylation of des- $\gamma$-carboxylated bone Gla protein and des- $\gamma$-carboxylated pro bone Gla protein. Biochemistry 34: 9541-9551, 1995.

17. Nakao M, Nishiuchi Y, Nakata M, Kimura T and Sakakibara S: Synthesis of human osteocalcins: $\gamma$-carboxyglutamic acid at position 17 is essential for a calcium-dependent conformational transition. Pept Res 7: 171-174, 1994.

18. Hannon R and Eastell R: Preanalytical variability of biochemical markers of bone turnover. Osteoporos Int 6: s30-s44, 2000

19. Hidaka K, Higashi M, Watanabe Y, Tomita M, Katsuyama H and Tamechika Y: An improved analytical method for detection of isoflavones in plasma by high performance liquid chromatography. Kawasaki Ikaishi Arts Sci 33: 9-15, 2007.

20. Tsangalis D, Wilcox G, Shah NP and Stojanovska L: Bioavailability of isflavone phytoestrogens in postmenopausal women consuming soya milk fermented with probiotic bifidobacteria. Br J Nutr 93: 867-877, 2005.
21. Hur HG, Lay JO Jr, Beger RD, Freeman JP and Rafii F: Isolation of human intestinal bacteria metabolizing the natural isoflavone glycosides daidzin and genistin. Arch Microbiol 174: 422-428, 2000

22. Atkinson C, Frankenfeld CL and Lampe JW: Gut bacterial metabolism of the soy isoflavone daidzein: exploring the relevance to human health. Exp Biol Med 230: 155-170, 2005.

23. Setchell KD: Absorption and metabolism of soy isoflavones from food to dietary supplements and adults to infants. J Nutr 130: 654s-655s, 2000.

24. Strand M, Soderstrom I, Wiklund PG, Hallmans G, Weinehall L, Soderberg S and Olsson T: Estrogen receptor alpha gene polymorphisms and first-ever intracerebral hemorrhage. Cerebrovasc Dis 24: 500-508, 2007.

25. Health and Welfare Statistics Association: J Health Welfare Stat. 55: 459, 2008. (In Japanese)

26. Mitra S, Desai M and Khatkhatay MI: Association of estrogen receptor $\alpha$ gene polymorphisms with bone mineral density in postmenopausal Indian women. Mol Genet Metab 87: 80-87, 2006.

27. Luukinen H, Kakonen SM, Petterson K, et al: Strong prediction of fractures among older adults by the ratio of carboxylated to total serum osteocalcin. J Bone Miner Res 15: 2473-2478, 2000.

28. Brink E, Coxam V, Robins S, Wahala K, Cassidy A and Branca F: Long-term consumption of isoflavone-enriched foods does not affect bone mineral density, bone metabolism, or hormonal status in early postmenopausal women: a randomized, double-blind, placebo controlled study. Am J Clin Nutr 87: 761-770, 2008.

29. Harkness LS, Fiedler K, Sehgal AR, Oravec D and Lerner E: Decreased bone resorption with soy isoflavone supplementation in postmenopausal women. J Womens Health 13: 1000-1007, 2004.

30. Branca F and Lorenzetti S: Health effects of phytoestrogens. In: Diet Diversification and Heath Promotion. Elmadfa I (ed). Vol. 57. Forum Nutr., Karger, Basel pp100-111, 2005.

31. Anderson JJB and Garner SC: The effect of phytoestrogens on bone. Nutr Res 17: 1617-1632, 1997. 